\title{
Dendritic cells infected with a vaccinia virus interleukin-2 vector secrete high levels of IL-2 and can become efficient antigen presenting cells that secrete high levels of the immunostimulatory cytokine IL-12
}

\author{
Sutapa Mukherjee, ${ }^{1}$ John W Upham, ${ }^{1}$ Ian Ramshaw, ${ }^{2}$ Christine Bundell, ${ }^{1}$ \\ Ivonne van Bruggen, ${ }^{1}$ Bruce WS Robinson, ${ }^{1,3}$ and Delia $J$ Nelson ${ }^{1,3,4}$
}

${ }^{1}$ Department of Medicine, University of Western Australia, Perth, Australia; ${ }^{2}$ Viral Engineering Group, Division of Cell Biology, John Curtin School of Medical Research, Australian National University, Canberra, Australia; ${ }^{3}$ Western Australian Institute for Medical Research, Perth, Australia; and ${ }^{4}$ School of Biomedical Sciences, Curtin University, Perth, Australia.

Dendritic cell (DC) therapies using DC presenting tumor antigen/s can induce CD8 ${ }^{+}$CTL that mediate tumor eradication, nonetheless many patients remain unresponsive. Thus, cytokine gene vectors applied to DC may amplify these responses. Herein, we examined the responses that monocyte-derived DC (at different maturational stages) make when infected with a vaccinia virusinterleukin-2 (VV-IL-2) vector in vitro. VV-IL-2-infected DC secreted significant levels of bioactive IL-2 and maintained their antigen presentation function. However, we show that DC are exquisitely sensitive to their local antigenic microenvironment, and that responses generated by one antigen can be altered by another. VV-IL-2 infection of immature DC led to DC activation (upregulation of CD80, CD86 and class II surface molecules) when the virus was propagated through xenogeneic, but not syngeneic, mammalian cells; these DC secreted IL-10 and tumor necrosis factor- $\alpha$ (TNF- $\alpha$ ), but not IL-12. In contrast, after VV-IL-2 infection (regardless of their mammalian cellular context), IFN $\gamma /$ LPS-matured DC inevitably downregulated their antigen presenting machinery. In conclusion, immunostimulatory DC can be generated by VV-IL-2, but this depends upon (i) infecting immature DC only, (ii) the mammalian cells through which the virus is prepared and (iii) individual donors; hence donors must be screened to assess their specific responses.

Cancer Gene Therapy (2003) 10, 591-602. doi:10.1038/sj.cgt.7700604

Keywords: Vaccinia virus vector; dendritic cells; IL-2; cytokine gene transfer

$I^{t}$ is becoming clear that while solid tumors are not hidden from the immune system, the host antitumor immune response is ineffective at preventing tumor development. ${ }^{1-3}$ As a result, a number of strategies have been used, including gene therapy, in an effort to boost antitumor immunity in cancer patients. We recently completed a phase I gene therapy trial in malignant mesothelioma (MM) patients involving intratumoral injections of a replication-restricted vaccinia virus-interleukin-2 (VV-IL-2) vector ${ }^{4}$. Vaccinia virus (VV) was chosen because its virology and safety are well described. Similarly, IL-2 was selected because it has demonstrated powerful effects in some animal models, ${ }^{5}$ and in human clinical trials, ${ }^{6-8}$ including MM. ${ }^{9,10}$ VV-IL-2 did not generate systemic or local

Received September 17, 2003.

Address correspondence and reprint requests to: Dr Delia Nelson Department of Medicine, University of Western Australia, 4th Floor G Block, QEII Medical Centre, Verdun Street, Nedlands, WA 6009, Australia.E-mail:delian@cyllene.uwa.edu.au toxicities, and despite an anti-VV response, VV-IL-2 mRNA gene expression persisted in tumor biopsies for up to 3 weeks inducing a local T-cell infiltrate suggesting that VV-IL-2 may be an ideal gene therapy vector for cancer patients.

In our study, no tumor regression was noted, which may reflect that the optimum therapeutic regimen has not yet been identified. Therefore, we examined the effects VV-IL-2 infection has on dendritic cells (DC), which are potent antigen presenting cells capable of activating naïve and memory T-cells, ${ }^{11}$ for two reasons. Firstly, after intratumoral injection, VV-IL-2 may infect tumor cells ${ }^{4}$ and tumor-infiltrating DC. These DC may transport tumor antigens to regional lymph nodes (LN) where they present these antigens to T-cells (a process referred to as cross-presentation, ${ }^{12,13}$ generating an antitumor response. Importantly, we have shown that MM tumor antigens are constitutively cross-presented in tumor-draining LN for the duration of tumor growth, ${ }^{2}$ but without "help" offered by $\mathrm{CD}^{+}{ }^{\mathrm{T}}$-cells ${ }^{14}$ or by $\mathrm{IL}-2$, the resulting immune response cannot restrain $\mathrm{MM}$ tumor growth. 
Hence, IL-2-expressing intratumoral DC may transport tumor antigens to the LN and offer "help" at the site it is most needed.

Secondly, DC-based therapies have emerged as a promising anticancer therapy; ${ }^{15,16}$ the rationale is that autologous DC are treated in vitro such that, after their return into patients, they evoke tumor-specific $\mathrm{CD}^{+}$ cytotoxic $\mathrm{T}$ lymphocytes (CTL) that eradicate tumors via the same mechanisms discussed above. Studies using DC treated with tumor antigens, including those expressed by viral vectors, have demonstrated the generation of tumorspecific immune responses that were occasionally associated with objective responses ${ }^{17-21}$ but, in general, the clinical outcomes have been disappointing; we speculated that these responses could be enhanced by expressing the IL-2 gene within DC. Thus, use of VV-IL-2-infected autologous DC may be an alternative to intratumoral VV-IL-2 injections.

The activation status of DC at the time of vaccination may be critical, and immature DC may inhibit rather than promote immunity. $^{22}$ Thus, mature DC that express high levels of class II and costimulatory molecules, and secrete IL-12, a cytokine critical for the generation of cellmediated immunity, rather than the immunoregulatory cytokine IL-10 are proposed to be the best vaccine candidates. Studies using wild-type, or recombinant, VV have demonstrated contradictory results; some studies report that $\mathrm{VV}$ infection modulates $\mathrm{DC}$ maturation reducing their antigen presentation capacity, ${ }^{23-25}$ while others show that VV-infected DC readily induce specific effector CTL. ${ }^{26-30}$ No studies, until now, have conducted a detailed examination of the effects an IL-2-encoding VV vector has on DC.

Ideally, VV-IL-2-infected DC will secrete IL-2, be activated, and traffic to tumor-draining $\mathrm{LN}$ providing sufficient IL-2 at the precise juncture when tumor antigens are presented to $\mathrm{CD}^{+} \mathrm{T}$-cells, thereby preventing T-cell anergy, ${ }^{14,31,32}$ and generating a potent, prolonged CTL response. As recent studies have shown that viral infection, or IL-2, can affect DC, we thought that it was important to understand the beneficial, or detrimental, effects VV-IL-2 infection has on DC. Therefore, these studies assessed the responses host DC (at different maturational stages) make during VV-IL-2 infection.

We show that DC are exquisitely sensitive to their local antigenic microenvironment, and that responses generated by one antigen can be altered by another. VV-IL-2 infection of immature DC induced production of significant amounts of bioactive IL-2, DC activation and maturation (upregulation of CD80, CD86 and class II), as well as maintenance of their antigen presentation capacity. However, these DC secreted IL-10 and tumor necrosis factor- $\alpha(\mathrm{TNF}-\alpha)$, but not IL-12. In contrast, mature DC downregulated CD80, CD86 and class II expression, but upregulated IL-12 secretion. However, these results were donor dependent and significantly influenced by the type of mammalian cell through which VV-IL-2 was passaged.

\section{Methods}

\section{Preparation of monocyte-derived DC}

Approval for the study was given by the Ethics Committee of the Sir Charles Gairdner Hospital, Perth, Western Australia. Peripheral blood mononuclear cells (PBMC) were isolated from healthy donors, aged 16-70 years, either from buffy coats or from $50 \mathrm{ml}$ of heparinized blood by Ficoll-Paque (Pharmacia Biotech, Uppsala, Sweden) density gradient centrifugation. DC were prepared using a modified procedure to that described. ${ }^{33}$ Except where indicated, cells were cultured in a complete medium containing LPS-free RPMI-1640 (Gibco BRL, Grand Island, NY), 10\% FCS (Invitrogen, Groningen, The Netherlands), $10 \mu \mathrm{g} / \mathrm{ml}$ polymixinB (Sigma), $48 \mathrm{mg} / 1$ gentamicin (Pharmacia \& Upjohn, Perth, Australia), $60 \mathrm{mg} / 1$ benzylpenicillin (CSL, Australia), $2 \mathrm{~g} / 1$ sodium hydrogen carbonate (BDH, Victoria, Australia), $20 \mathrm{mM} \quad N$-2-hydro-2ethylpiperazine- $N$-2-ethane sulfonic acid (Hepes; Gibco BRL) and $5 \times 10^{-5} \mathrm{M}$ 2-mercapto ethanol (Sigma).

Briefly, $10^{8}$ PBMC were allowed to adhere to Falcon T75 culture flasks (Becton Dickinson, Mountain View, $\mathrm{CA}$ ) in complete medium. After 2 hours at $37^{\circ} \mathrm{C}$, nonadherent cells were removed, and the remaining adherent cells were cultured for 7 days with $50 \mathrm{ng} / \mathrm{ml}$ GM-CSF (RD Systems, Minneapolis, MN) and $10 \mathrm{ng} / \mathrm{ml}$ IL-4 (RD Systems). Cultures were supplemented with cytokines and media on days 3 and $6 . \mathrm{CD}^{+} \mathrm{DC}$ expressing low levels of class II, and little or no CD80 or CD86 were considered to be immature (data not shown). To ensure that we routinely obtained DC expressing this phenotype, DC were continuously cultured in an LPS-free environment.

In some experiments, DC were primed with $20 \mathrm{ng} / \mathrm{ml}$ IFN $\gamma$ (Boehringer Mannheim, Indianapolis, IN) for 3-4 hours prior to the addition of other agents such as $10 \mu \mathrm{g} /$ $\mathrm{ml}$ lipopolysaccharide (LPS, Sigma) and/or the VV constructs.

\section{Cell lines}

The $143 \mathrm{BTK}^{-}$cell line (referred to as 143B), a human fibroblast osteosarcoma cell line lacking the thymidine kinase (TK) gene (kindly donated by Dr Jane Allan, Department of Microbiology, University of WA, Australia), was maintained in minimum essential media (MEM) with L-glutamine (GIBCO BRL, NY) supplemented with $10 \% \mathrm{FCS}$, gentamicin and benzylpenicillin, and cultured at $37^{\circ} \mathrm{C}$ in $5 \% \mathrm{CO}_{2}$.

\section{$V V$-IL-2 and the control $V V$}

The recombinant VV expressing IL-2 (VV-IL-2) was constructed by isolating a $1 \mathrm{~kb}$ XhoI from pcD-IL-2 (KI Arai, DNAX Research Institute, Palo Alto, CA). ${ }^{34} \mathrm{~A}$ $500 \mathrm{bp}$ human IL-2 cDNA was inserted into plasmid pBCB07. This plasmid was introduced into the thymidine kinase (TK) gene in the $\mathrm{J}$ region of the $\mathrm{NYCBOH}$ strain of VV. TK-negative recombinant viruses were selected for IL-2 expression. ${ }^{34}$ 
The control (noncytokine expressing) virus vector (kindly supplied by Dr Jane Allan, Department of Medicine, University of Western Australia) consisted of VV encoding an immunodominant epitope, YPHFMPTNL, of murine cytomegalovirus pp89.

\section{Propagation of recombinant $V V$ constructs}

The VV constructs were propagated in two ways. Firstly, confluent flasks of human fibroblast osteosarcoma 143B cells were infected with approximately $10^{9}$ plaque-forming units (PFUS). After 3 days, cells were removed, resuspended in MEM $/ 2.5 \%$ FCS, lysed by three freeze-thaw cycles, and stored at $-70^{\circ} \mathrm{C}$. Secondly, under conditions appropriate to the Therapeutic Goods Act, the 143B cell line was initially used to produce VV-IL-2, which was then plaque purified and propagated in fibroblasts harvested from specific pathogen-free chicken embryos.

\section{$V V$ infection of $D C$}

$\mathrm{VV}$ constructs were added at varying concentrations to $10^{6}$ cultured $\mathrm{DC} / \mathrm{ml}$ and left for $16-48$ hours at $37^{\circ} \mathrm{C}$ and $5 \% \mathrm{CO}_{2}$. Culture supernatants were collected for cytokine measurement, and DC were washed and used in the experiments outlined below. In control experiments, DC were exposed to media, LPS with or without IFN $\gamma$ or recombinant human IL-2 (Cetus Corporation, Emeryville, CA).

\section{Plaque-forming assay}

A plaque-forming assay was used to determine viral concentrations. Virus constructs were added to confluent 143BT cells and incubated in MEM $/ 2.5 \%$ FCS for 2 days, after which the medium was removed and $0.5 \mathrm{ml}$ of $0.1 \%$ crystal violet (Sigma, St Louis, MO) in 20\% ethanol was added for 5 minutes at RT and plaques were counted.

\section{Allogeneic mixed lymphocyte response}

Nonadherent PBMCs from blood samples obtained from healthy donors were used as the source of responder lymphocytes. Varying numbers of DC were cocultured with $2 \times 10^{5}$ responder such that the DC:responder cell ratio ranged from $1: 810$ to $1: 10$, in complete medium, without polymixinB, IL-4 or GM-CSF. Plates were labeled with $1 \mu \mathrm{Ci}\left[{ }^{3} \mathrm{H}\right]$ thymidine, harvested 18 hours later, and DNA synthesis was measured by liquid scintillation counting (Hewlett Packard, Palo Alto, CA).

\section{Immunohistochemistry and FACS analysis}

Cell surface markers were analyzed by flow cytometry. Cells were incubated for 30 minutes at $4{ }^{\circ} \mathrm{C}$ with the following monoclonal antibodies (mAbs) diluted in PBS with $0.1 \%$ azide and $5 \%$ normal mouse serum (NMS): CD1a-FITC, CD80-PE, CD86-PE, HLA-DR-cychrome C, CD-25PE (all from Pharmingen, San Diego, CA). The cells were then washed and fixed in phosphate-buffered saline supplemented with $1 \%$ paraformaldehyde. In all, 10,000 events were acquired and analysis was performed on a FACScan (Becton Dickinson, Mountain View, CA) using Cell Quest software. The proportion of cells expressing CD1a, as a marker of DC purity, averaged $84 \pm 11 \%$ (data not shown).

In order to determine the proportion of DC infected with VV-IL-2, cytospins of washed DC were prepared using a Shandon II cytocentrifuge (Shandon Lipshaw, Pittsburgh, PA) set at $800 \mathrm{rpm}$ for 5 minutes, fixed with cold ethanol $(100 \%)$, blocked with $1 \%$ BSA in PBS, incubated with rabbit anti-VV, and visualized using goat anti-rabbit conjugated to FITC with $25 \mu \mathrm{g} / \mathrm{ml}$ propidium iodide (PI; Sigma) in 1\% BSA and $0.5 \%$ human IgG. Slides were mounted and sealed using $25 \%$ glycerol, $18 \%$ polyvinyl alcohol (PVA) in Tris buffer.

\section{Cytokine assays}

Quantitation of IL-2, IL-12p70, IL-10 and TNF $\alpha$ from DC supernatants collected 18-24 hours after treatment (as described in results) was performed using commercial ELISA kits (Pharmingen, San Diego, CA).

\section{Determining IL-2 bioactivity}

The bioactivity of IL-2 secreted from VV-IL-2-infected DC was assayed using a murine IL-2-dependent cytotoxic T-cell line (CTLL), as previously described. ${ }^{35}$ Briefly, $10^{4}$ CTLL cells/well/ $50 \mu \mathrm{l}$ were plated with $50 \mu \mathrm{l}$ of varying concentrations of test supernatant. CTLL proliferation was determined 24 hours later by $\left[{ }^{3} \mathrm{H}\right]$ thymidine incorporation (for 5 hours) and measured by liquid scintillation counting (Hewlett Packard, Palo Alto, CA). Activity is expressed as the median effective dose $\left(\mathrm{ED}_{50} ; \mathrm{U} / \mathrm{ml}\right)$, where $1 \mathrm{U}$ is the amount required for $50 \%$ maximum growth from $1 \times 10^{4}$ CTLL cells in $0.1 \mathrm{ml}$ culture volumes during a 24-hour culture period.

\section{Statistical analysis}

One-way analysis of variance (ANOVA) was used to compare cytokine production from VV-IL-2-infected DC, or the mean ${ }^{3} \mathrm{H}$-thymidine incorporation, within each group across the different groups. Minitab for Windows v12.1 (Minitab Inc.) was used to manage and analyze the data. Statistical significance was defined at the standard $5 \%$ level.

\section{Results}

Immature DC are infected by VV-IL-2 and secrete bioactive IL-2

As immature DC are efficient at antigen capture, the first series of experiments sought to determine whether VV-IL2 could infect immature DC by determining the efficiency of infection (the transduction efficiency) of the virus for DC. We used VV-IL-2 virus in a chicken fibroblast lysate, as this viral preparation was administered intratumorally into MM patients in our clinical trial. ${ }^{4}$

Immature DC were infected with VV-IL-2 for 18-24 hours and (i) visualized by immunostaining using anti-VV antibodies (Fig 1a), or (ii) placed in a plaque-forming 

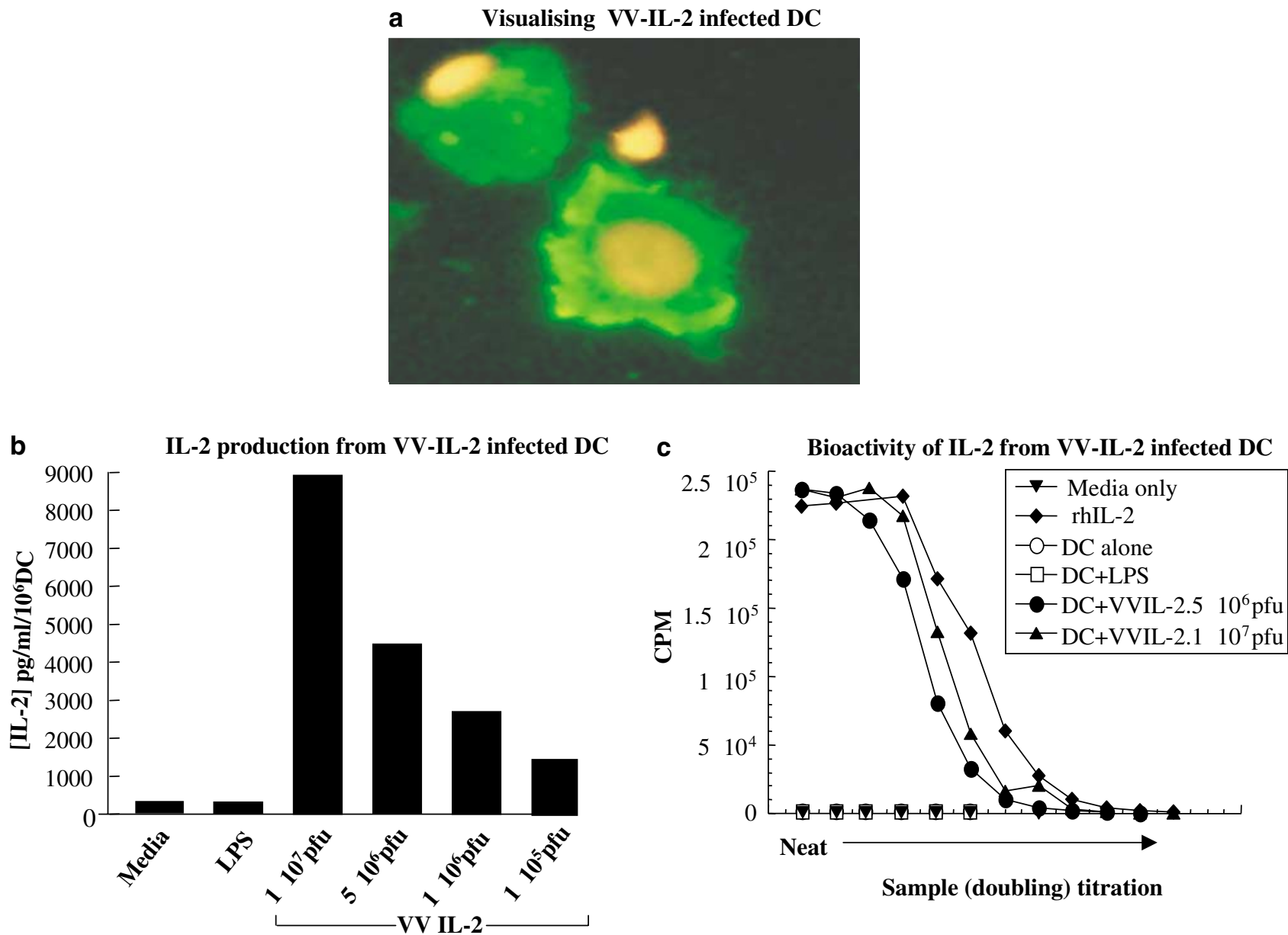

Figure $1 \mathrm{VV}$-IL-2-infected monocyte-derived DC produce bioactive IL-2. DC were infected with different concentrations of VV-IL-2 for 24 hours. (a) DC infected with $10^{7}$ PFU VV-IL-2 and stained with an anti-VV antibody (green) and PI (orange showing the nucleus). (b) IL-2 production in culture supernatants measured by ELISA. Controls include recombinant human IL-2, DC left untouched (media), or stimulated with LPS. (c) IL-2 from infected DC stimulated CTLL proliferation.

assay on 143B cells (data not shown) and the numbers of plaques versus DC determined; in both systems, the transduction efficiency was determined to be $30 \%$ at an m.o.i. of 1 .

Infected immature DC produced significant amounts of IL-2 (Fig 1b). A dose-response was observed with the highest level of IL-2 produced by DC infected with the highest concentration of VV-IL-2 $\left(1 \times 10^{7}\right.$ PFU; m.o.i of 10). DC incubated with media alone or LPS produced minimal amounts of IL-2. The IL-2 produced from VVIL-2-exposed DC was shown to be bioactive when used in a CTLL assay (Fig 1c).

$V V$-IL-2 infection stimulates immature monocytederived DC to express a mature phenotype

To determine whether VV-IL-2 infection alters the expression of molecules that are critical for antigen presentation and $\mathrm{T}$-cell activation, immature $\mathrm{CD} 1 \mathrm{a}^{+}$ DC (expressing low levels of class II, and little or no CD80 or CD86) were infected with different concentrations of VV-IL-2 and surface marker expression was determined. VV-IL-2-infected DC were compared to uninfected, media alone, controls. After exposure for 24 hours to VV-IL-2 ( $\left.10^{7} \mathrm{PFU}\right), 100 \%$ of immature DC prepared from different donors showed significant surface upregulation of the costimulatory molecule B7.2 (CD86; seen in a representative example in Fig 2a, and pooled data in Fig 2d). This response was dose dependent; DC exposed to the highest concentration of VV-IL-2 ( $10^{7}$ PFU) demonstrated CD86 expression levels similar to those seen in LPS-stimulated DC.

In contrast to the consistent effects that VV-IL-2 exerted upon DC expression of CD86, greater donor variation was seen with MHC Class II (HLA-DR; Fig 2d) and CD80 (Fig 2d). In 50\% of donors HLA-DR expression was unchanged, while in the remaining donors HLA-DR expression increased (Fig 2b). CD80 expression varied in different donors (Fig 2c). In individuals where an increase in CD80 expression was seen, the upregulation was dose dependent (Fig 2d).

As this work used a viral construct coding for IL-2, expression levels of CD25 (the $\alpha$ chain of the IL-2R) on 


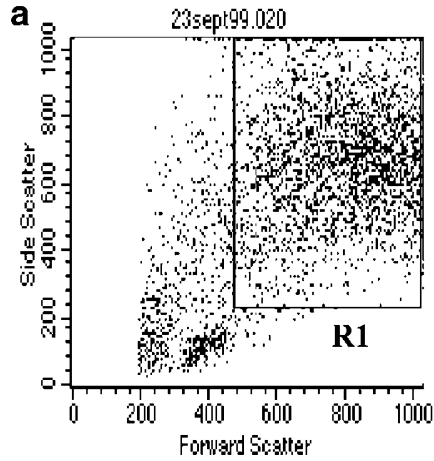

b
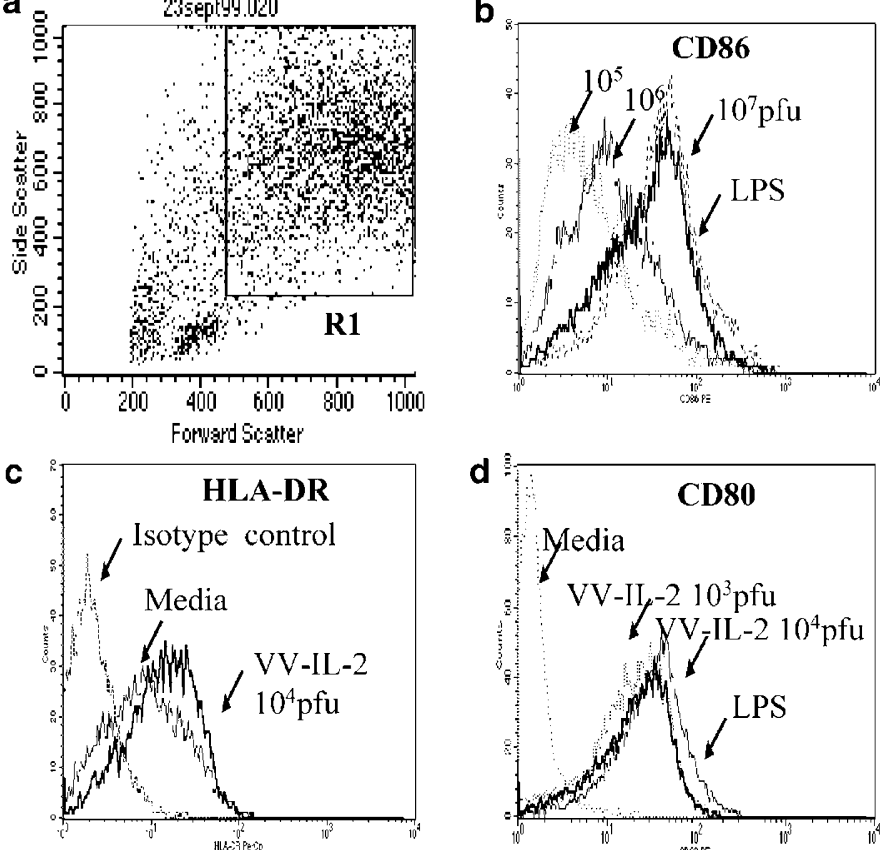

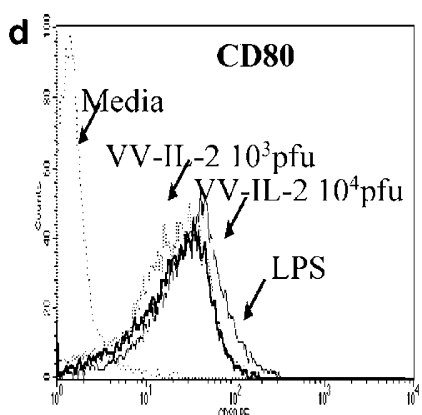

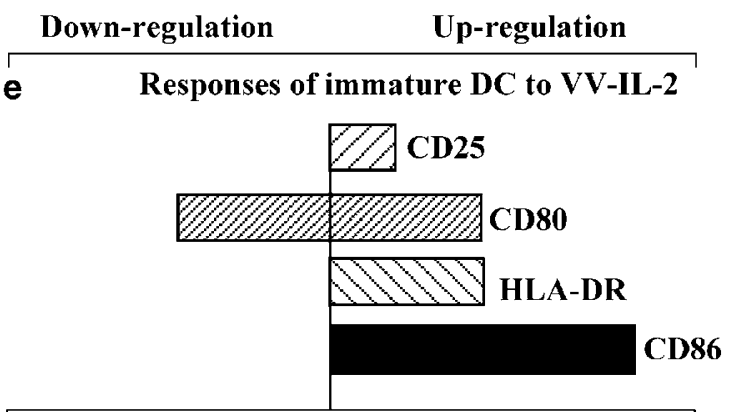

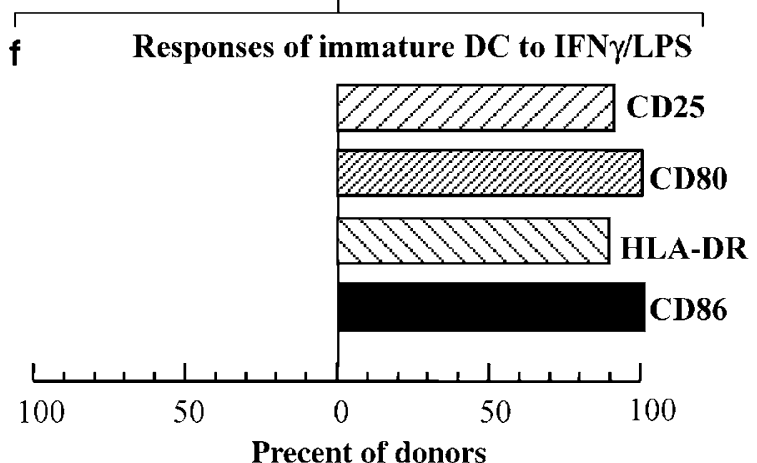

Figure 2 Immature DC infected with V-IL-2 (propagated in chicken fibroblasts) generally express an activated phenotype. Immature DC were exposed to LPS or different concentrations of VV-IL-2 for 16-24 hours and their maturation analyzed by FACS analysis.(a-c) FACS staining of a representative example of eight different donors.(b) Some donor variation was seen in response to VV-IL-2 (d), but not to LPS/IFN $\gamma$ stimulation (e).

VV-IL-2-infected DC were also examined. CD25 expression was not altered in $75 \%$ of donors, while in the remaining donors a slight increase was seen (Fig 2d).

To assess whether there were any intrinsic differences between DC from different donors that could explain the data described above, LPS/IFN $\gamma$ was used as a positive control. In contrast to the variable nature of immature DC responses to VV-IL-2, a reproducible and robust response was seen when DC were 'primed' with IFN $\gamma$ for 3-4 hours before the addition of LPS. A highly activated population of mature DC (Fig 2e) was generated that expressed increased levels of CD86, CD80, HLA-DR and CD25.

\section{$V V$-IL-2 infection does not inhibit the antigen presenting function of $D C$}

To assess the functional consequences of infection with VV-IL-2, we evaluated the ability of VV-IL-2-infected immature DC to stimulate allogeneic T-cells. DC infected with VV-IL-2 produced an allogeneic T-cell response that was not significantly different from LPS-stimulated DC (Fig 3); that is, we saw no evidence that infection with VV-IL-2 led to impaired antigen presentation. This was true regardless of the day the plates were labeled with ${ }^{3} \mathrm{H}$ (i.e. days 4, 5, 6 or 7 of culture; data not shown).

$V V$-IL-2 infection alters the surface phenotype of mature LPS/IFN $\gamma$-treated DC

The data above demonstrated that VV-IL-2-exposed immature DC generally assumed a (more) mature phenotype. However, others have shown that wild-type, or recombinant, $\mathrm{VV}$ inhibits $\mathrm{DC}$ maturation of one or more of the molecules examined here. ${ }^{23-25}$ Therefore, the next series of experiments examined the consequences of VV-IL-2 infection of IFN $\gamma$ LPS-matured DC. VV-IL-2 was added to IFN $\gamma$ "primed" DC at the same time as LPS. The data are shown as a comparison of IFN $\gamma /$ LPSmatured DC versus the same DC coexposed to VV-IL-2.

CD86 expression was inhibited, or downregulated, in $75 \%$ of donors (a representative sample is shown in Fig 4a; pooled data in Fig 4e); the levels varied from a minor change to a 3-4 log fold reduction. HLA-DR and CD80 levels were also reduced in 100 and $40 \%$ at donors, respectively (Fig 4b and c, respectively, and Fig 4e). In the latter case, the reduction was not dramatic (up to a $0.5 \mathrm{log}$ fold reduction) and $60 \%$ of donors did not alter their HLA-DR surface expression levels. Interestingly, CD25 surface expression increased in the majority of donors; Fig 4d and e). Collectively, these data suggest that VV-IL2 infection of mature DC generally downregulates IFN $\gamma /$ LPS-driven expression of molecules on DC that are critical for the activation of naïve T-cells.

\section{$V$-IL-2-exposed DC secrete $I L-10$ and $T N F \alpha$ but not IL-12}

DC can release a number of cytokines that influence T-cell differentiation. IL-12 appears to be critical for cell-mediated immunity, $\mathrm{TNF} \alpha$ is a proinflammatory cytokine, while IL-10 has important immunoregulatory properties that may be important in the generation of 


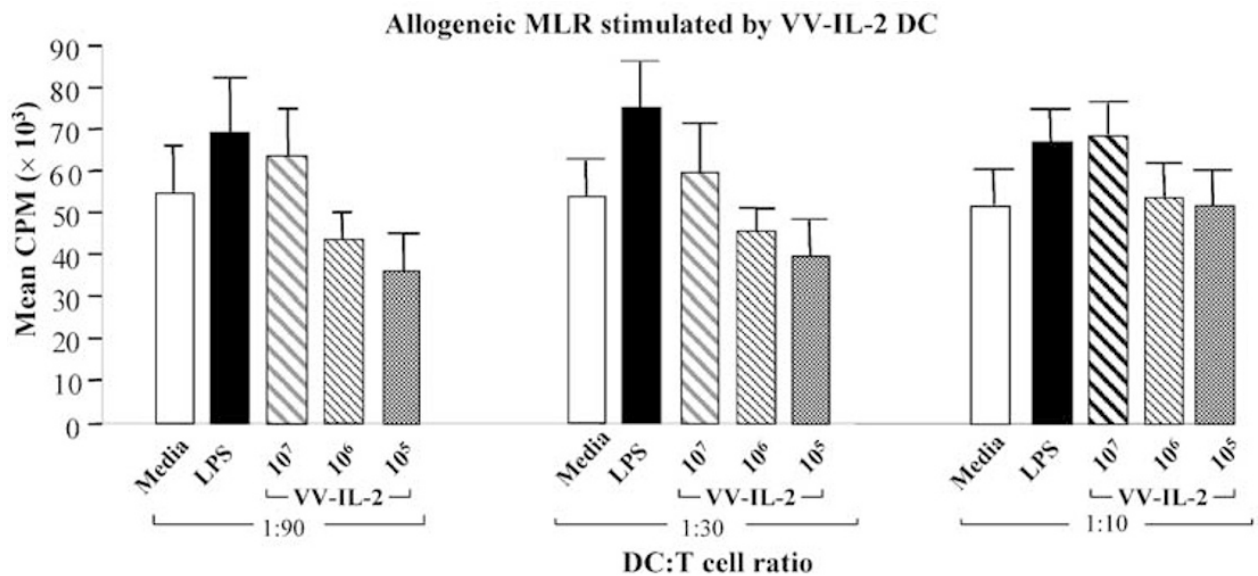

Figure 3 VV-IL-2 infection does not impair the capacity of DC to present antigen. After washing, DC (treated as per Fig 2) were added in graded doses to $2 \times 10^{5}$ allogeneic T-cells. After 6 days, T-cell proliferation was assessed by ${ }^{3} \mathrm{H}$-thymidine incorporation and shown as the mean $\pm \mathrm{SD}$ of five independent experiments with different donors.
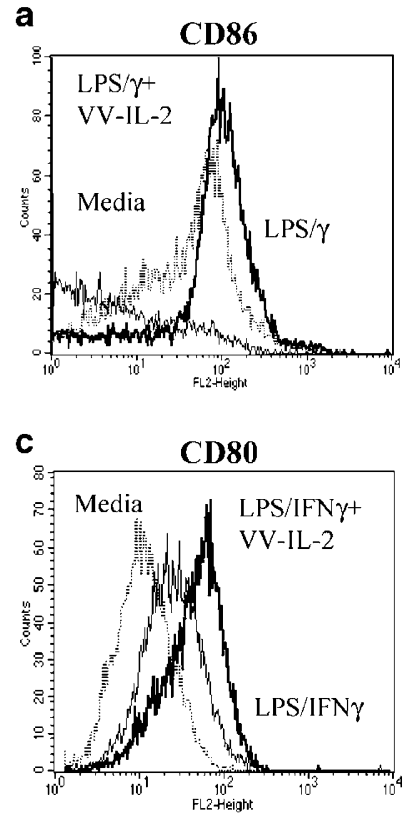
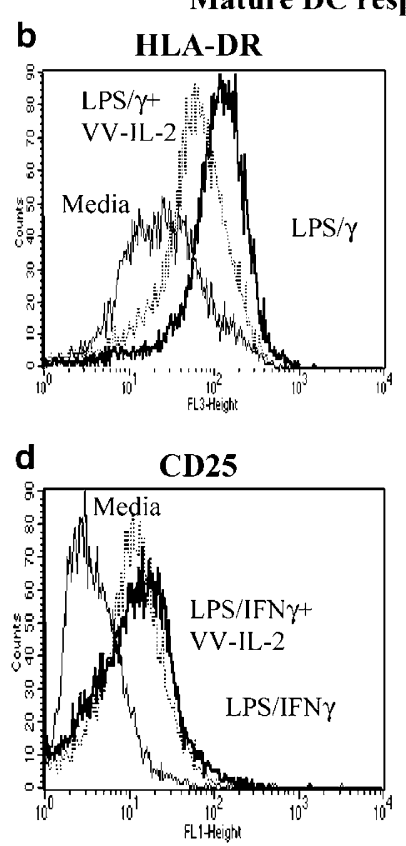

e Down-regulation Up-regulation Response of mature DC to VV-IL-2

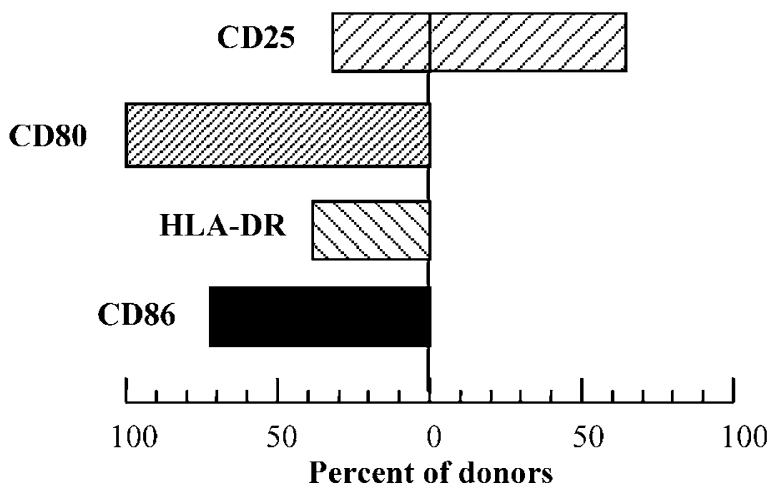

Figure 4 VV-IL-2 downregulates activation markers in LPS/IFN $\gamma$-matured DC. DC were primed with IFN $\gamma$ on day 7 of culture for 3-4 hours, costimulated with LPS and $10^{7}$ PFUS VV-IL-2 and surface expression of activation markers was examined by FACS analysis. (a-d): FACS staining (as indicated) of a representative example. (e) Variation of donor responses.

immune tolerance. Accordingly, the effects of VV-IL-2 on the ability of DC to produce these cytokines were tested. Immature DC infected with VV-IL-2 produced a dose-dependent increase in production of both IL-10 and $\mathrm{TNF} \alpha$ (Figs 5a and b, respectively), but even the highest viral load did not generate the levels seen in IFN $\gamma /$ LPSexposed DC.

Only trace amounts of IL-12p70 were produced by immature DC, or DC infected with varying doses of VVIL-2 for 24 hours (Fig 5c) or 48 hours (data not shown). DC matured only with LPS (i.e. without IFN $\gamma$ priming) and VV-IL-2-infected DC that had been primed with IFN $\gamma$ (but not stimulated with LPS) did not secrete IL-12 at either time point (data not shown). In contrast, high levels of IL-12 were produced from control IFN $\gamma /$ LPS-treated DC (Fig 5c).

Unexpectedly, IFN $\gamma /$ LPS-matured DC cotreated with VV-IL-2 further increased IL-12 production relative to IFN $\gamma /$ LPS-only-mature DC (Fig 5d). In contrast, IL-10 secretion from these mature DC did not change after exposure to VV-IL-2 (Fig 5d).

The data above show that VV-IL-2 infection can modulate DC phenotype, and the pattern of cytokines they produce, but does not alter the antigen presenting 

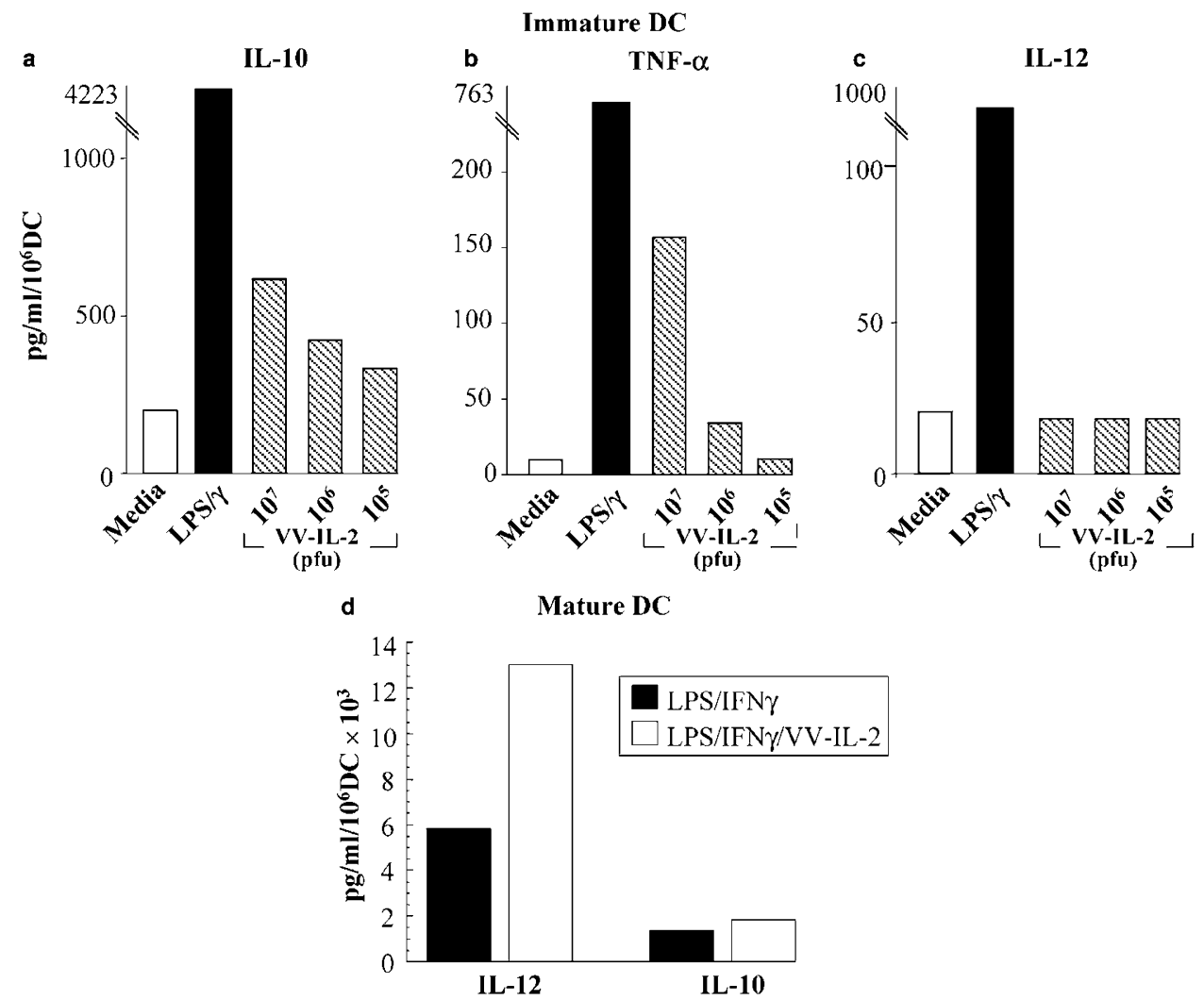

Figure 5 Contrasting IL-12 responses in VV-IL-2-infected immature versus mature DC. DC were cultured for 24 hours in media alone, LPS/IFN VV-IL-2 $\left(10^{7}, 10^{6}\right.$ or $\left.10^{5} \mathrm{PFU}\right)$ and cytokine concentrations were measured in the resulting supernatants. Infected immature DC produced IL-10 (a), TNF $\alpha$ (b), but not IL-12 p70 (c). In contrast, mature IFN $\gamma /$ LPS-stimulated DC infected with VV-IL-2 secreted higher levels of IL-12, but not IL10 (d).

function of DC. However, the VV-IL-2 preparation used in these initial experiments consisted not only of viral particles, but also cellular debris, and infected DC secreted IL-2. Thus, the next series of experiments evaluated the effect each of these separate components has on DC.

\section{Exogenous IL-2 has no effect on the phenotype or function of immature or mature $D C$}

In order to determine the effect of exogenous IL-2, varying doses of IL-2 alone were added to immature and IFN $\gamma /$ LPS-matured DC 24 hours prior to FACS analysis. The IL-2 doses were based upon the levels secreted by VV-IL-2-infected DC and ranged from 100 to $2 \mathrm{ng} / \mathrm{ml}$ (see Fig 1). In general, the expression levels of CD86, HLADR and CD25 of IL-2-treated immature DC were not different from those seen for the media only immature DC controls (data not shown). Similarly, the phenotype of mature DC remained unchanged after exposure to exogenous IL-2 (data not shown).

In one series of experiments, an excess of exogenous IL2 (2000, 200 and $20 \mathrm{ng} / \mathrm{ml})$ was added to DC for overnight culture. After washing, these DC were placed in an MLR assay. ANOVA analysis showed no difference between DC groups at all DC:T cell ratios tested (all p-values $>0.96$; data not shown). Hence, addition of exogenous
IL-2 does not appear to alter DC function or phenotype significantly.

Cellular lysates can alter the phenotype of immature and mature $D C$

Embryo-derived fibroblasts from SPF chickens are not readily available; therefore we prepared lysates from the human 143B osteosarcoma cell line which is generally used to propagate $\mathrm{VV}$, and examined the effects of these lysates on DC. It was estimated that $10^{7} \mathrm{PFU}$ of virus stock could maximally consist of $3 \times 10^{6}$ lysed 143 B cells. Varying doses of the cellular lysate were added to $10^{6}$ immature or IFN $\gamma /$ LPS-matured DC and their phenotype determined 24 hours later.

Responses to 143B cellular lysate were consistent between mature and immature DC populations for CD25 and HLA-DR expression. CD25 expression was not significantly altered in immature (Fig 6a), or mature DC (Fig 6b). HLA-DR expression was generally down regulated in immature and mature DC (Figs 6a and b); however, these changes represented only slight shifts in staining intensity (data not shown).

Interestingly, expression of both costimulatory molecules (CD80 and CD86) was significantly upregulated in immature, lysate-exposed DC (Fig 6a). In contrast, for mature, lysate-treated, DC, CD 80 was either slightly 
a

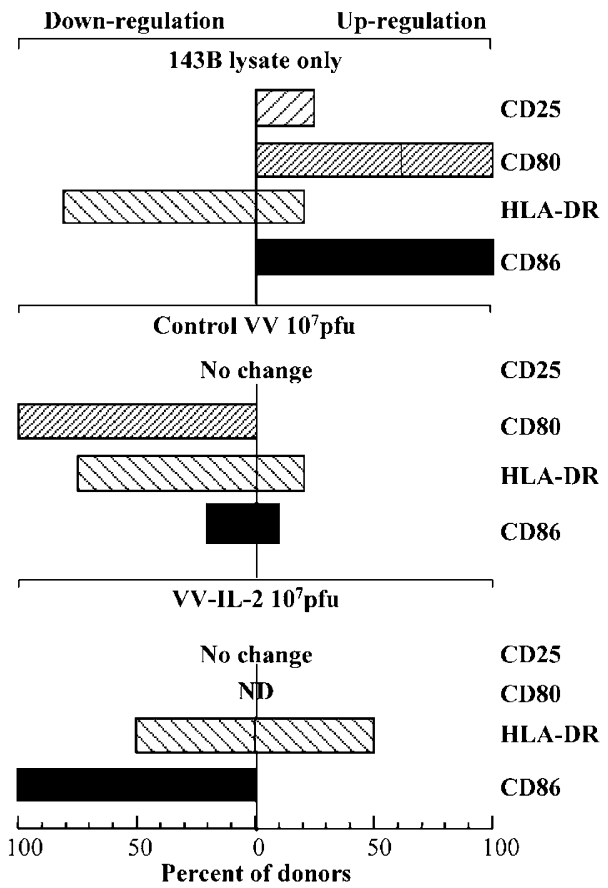

b Mature DC

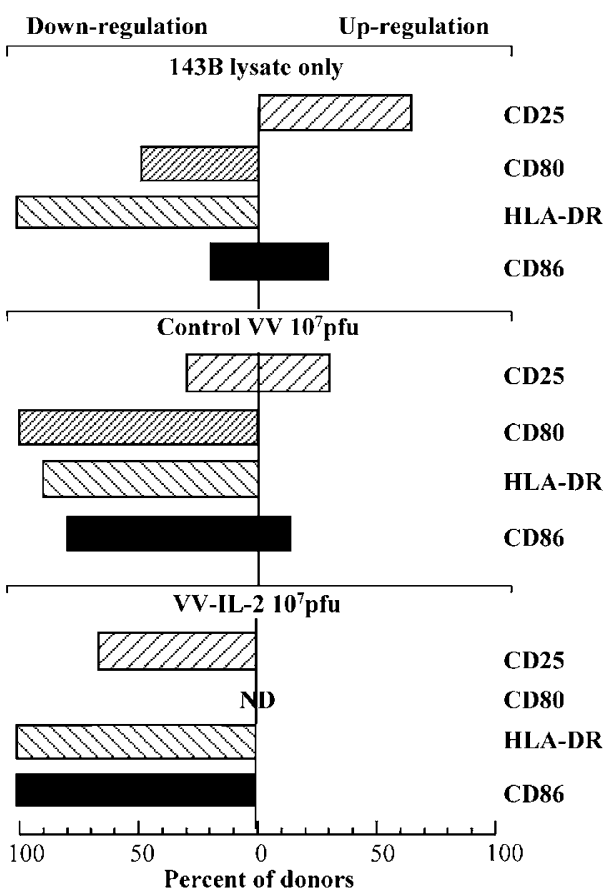

Figure 6 Variable DC responses to lysate only versus the VV constructs in the same lysate. Immature (a) and IFN $\gamma / \mathrm{LPS}$-matured (b) DC were given 143B lysate only, the control VV in 143B lysate, or VV-IL-2 in 143B lysate and their expression levels of CD25, CD80. HLA-DR and CD86 were compared.

downregulated or unaltered. Greater donor variation was seen with CD86 expression (Fig 6b); CD86 was weakly upregulated, not changed, or significantly (2.5 log fold) downregulated (Fig 6b and data not shown).

A noncytokine, control $V V$ presented in the same $143 B$ cellular lysate induces a different DC phenotype to DC given the lysate only

A control VV (noncytokine encoding vector) propagated in $143 \mathrm{~B}$ cells was used to infect immature and mature DC. In contrast to the results seen in lysate-only-treated DC, CD86 expression levels on immature DC were not altered in the majority of donors (Figs 6a and 7) after exposure to the control VV. In agreement with others using VV, CD80 was downregulated in $100 \%$ of donors, ${ }^{25,26}$ that is, the opposite response seen when immature DC were treated with lysate only (Fig 6a) or with VV-IL-2 (Fig 2). Similarly, HLA-DR was generally downregulated on immature DC at the highest VV concentration used (Fig 6a).

Mature DC (Fig 6b) exposed to the control VV generally decreased their expression levels of CD86 (see also Fig 7), CD80 and HLA-DR relative to DC stimulated only with $\mathrm{LPS} / \mathrm{IFN} \gamma$, although some donor variation was noted. CD25 responses on mature DC were highly inconsistent; reduced expression was seen in $44 \%$ of donors $(n=9)$, unchanged or slightly elevated in the remaining donors. These data imply that signals delivered by the lysate are altered by the presence of VV viral particles.

$V$ V-IL-2 in different cellular lysates generate phenotypically different DC

The VV-IL-2 construct was also propagated in 143B cells. Immature and LPS/IFN $\gamma$-matured DC were exposed to $10^{7} \mathrm{PFU}$ of this viral preparation. In contrast to the VV-IL-2 prepared in chicken fibroblasts, CD25 expression on immature DC remained unchanged, and CD86 was inevitably slightly reduced (Figs 6a and 7). HLA-DR expression varied in different donors from increased to decreased expression (Fig 6).

In mature DC (Fig 6b), VV-IL-2 in 143B lysate induced a similar response to that seen when the same virus was propagated in chicken fibroblasts, although less donor variation was seen with the former preparation; that is, downregulation of all markers examined. In some donors, CD86 expression decreased by 4 logs (Fig 7 and data not shown). HLA-DR expression was weakly downregulated while CD25 expression decreased or remained unaltered when exposed to VV-IL-2 in 143B lysate; in contrast, some donors increased CD25 after exposure to VV-IL-2 in the fibroblast lysate (Fig 6b).

IL-12 secretion by mature DC is dependent upon the cellular context of the virus

IL-12 secretion was not induced by immature DC infected with VV-IL-2 in either lysate (data not shown and Fig 5) 
CD86

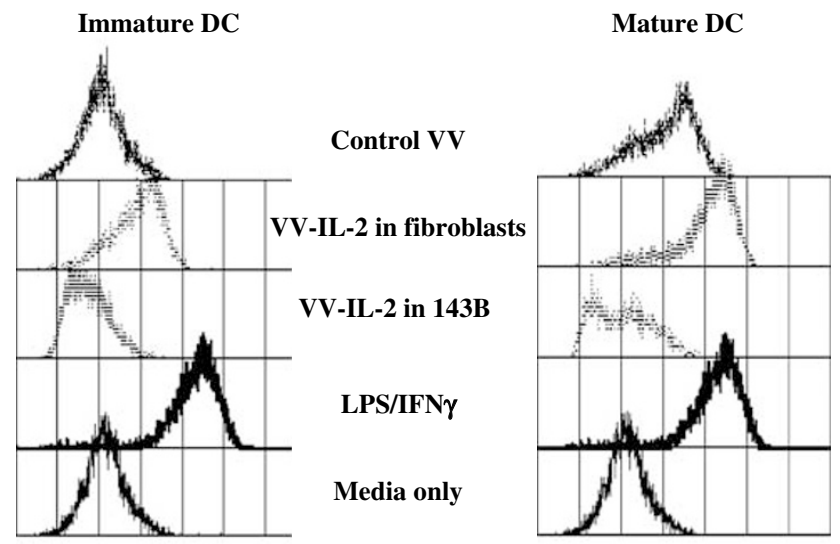

Figure 7 Differential CD86 responses to VV-IL-2 in different lysates. Immature (LHS) and IFN $\gamma /$ LPS-matured (RHS) DC were given the control VV in 143B lysate, VV-IL-2 in 143B lysate, or VV-IL-2 in chicken fibroblast lysate and their CD86 expression levels were compared.

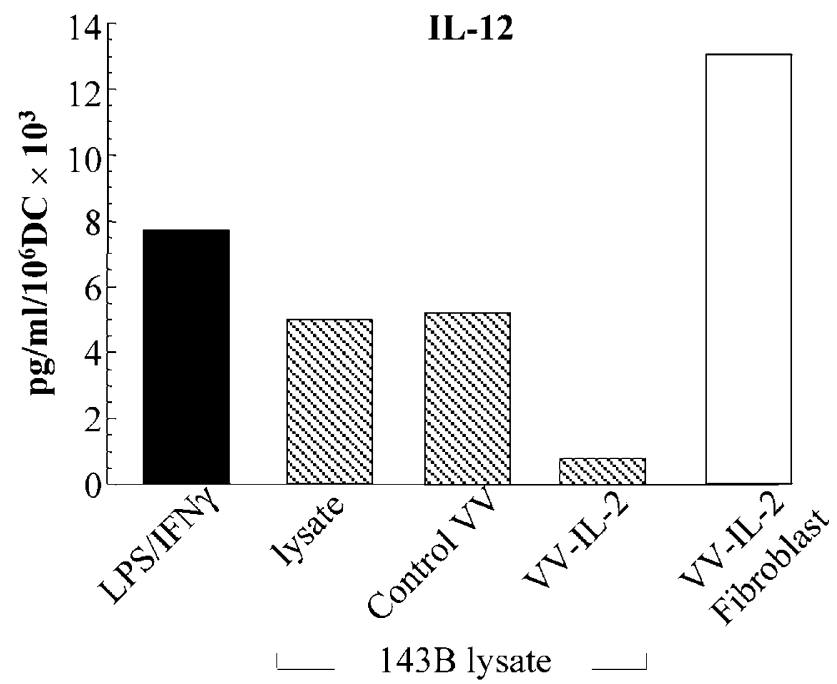

Figure 8 Differential IL-12 production from mature DC infected with VV-IL-2 in different lysates. LPS/IFN $\gamma$ matured DC were cultured for 24 hours in media alone, $10^{7}$ PFU of the control VV or VV-IL-2 in 143B lysate, $10^{7} \mathrm{PFU}$ of VV-IL-2 in chicken fibroblast lysate or ' $10^{7}$ PFU' equivalent of the 143B lysate only. IL-12 was measured in the supernatants 24 hours later.

or by the control VV (data not shown). However, IL-12 responses in mature DC differed greatly depending upon the cellular context the virus was propagated in. As expected, IFN $\gamma /$ LPS-matured DC secreted high levels of IL-12p70. Coexposure of IFN $\gamma /$ LPS-matured DC with the 143B lysate alone or the control VV (also in 143B lysate) resulted in slightly reduced IL-12 secretion (Fig 8). Mature DC treated with VV-IL-2 in lysed chicken fibroblasts inevitably increased IL-12 production (Fig 8), while (unexpectedly) the same VV-IL-2 construct prepared in lysed 143B cells significantly reduced IL-12 production to near zero levels (also in $100 \%$ of donors).

\section{Discussion}

While many current anticancer immunization regimens are able to induce immune responses, they are generally insufficient to induce tumor regression. One possible approach to this dilemma is to manipulate DC with cytokine genes in the hope that this may lead to an altered cytokine microenvironment within secondary lymphoid tissue that directly, or indirectly, enhances tumor-specific cytotoxic T-cell responses. As VV appears to be an ideal vector that can replicate in vivo without inducing adverse effects ${ }^{4}$ and has been successfully used to transduce DC with tumor antigens, ${ }^{26,28-30,36-39}$ such that these DC induce antigen-specific CTL, we examined the effects an IL-2-encoding, replication-deficient, VV vector has on DC.

We chose IL-2 because immunologic manipulation of tumor-bearing hosts with IL-2 can, occasionally, lead to complete rejection of cancer by promoting CTL function. ${ }^{40}$ Similarly, tumour cell lysate-pulsed DC have demonstrated enhanced antitumor therapeutic efficacy in vivo when combined with the systemic administration of IL-2. ${ }^{41-43}$ Furthermore, DC possess IL-2 receptors, and IL-2 stimulation of CD25 (IL-2R $\alpha$ ) may play a role in antigen uptake and affect DC maturation. ${ }^{44}$

\section{$V V$-IL-2 infected DC secrete IL-2 and maintain their antigen presenting function}

Our study commenced with an examination of the responses monocyte-derived DC make to the same VV preparation used in our human gene therapy clinical trial, in which $10^{7}$ PFUS of VV-IL-2 were given intratumorally to MM patients. ${ }^{4}$ This viral preparation was prepared in a manner that satisfied the Therapeutic Goods Act, that is, in SPF chicken embryo fibroblasts. VV-IL-2-infected immature and LPS-matured DC, leading to the production of significant amounts of bioactive IL-2. IL-2 secretion was dose dependent and infected DC remained viable for at least 48 hours.

Studies using wild-type VV, or recombinant VV, found that VV infection did not lead to DC maturation. ${ }^{23-25}$ These studies reported contradictory findings on the ability of VV-infected DC to stimulate T-cells. However, VV is highly immunogenic; and others have shown that VV-infected DC readily induced functional CTL responses. ${ }^{28-30,40}$. Similarly, we found that DC infected with high VV-IL-2 doses were at least as capable as uninfected, LPS-matured DC to stimulate allogeneic Tcell proliferation.

\section{The cellular context $V V$-IL-2 is prepared in} alters the $D C$ response

In two of the studies discussed above, VV infection selectively reduced expression of the costimulatory molecule CD80, while other surface molecules including CD86 and MHC class II remained unchanged. After infection with VV-IL-2 (in chicken fibroblasts), immature DC demonstrated variable responses with class II and CD80 expression, which were either upregulated or 
unaltered in different donors. In contrast, CD86 was inevitably upregulated in a dose-dependent manner reaching expression levels similar to those seen on LPSexposed DC. However, propagating the same viral vector in a different mammalian cellular context, the human fibroblast osteosarcoma (143B) cell line generated DC with a different phenotype; CD86 expression was never upregulated in immature DC, while HLA-DR was either downregulated or unaltered after exposure to VV-IL-2 in the 143B lysate. These data may explain the variable reports seen in the literature and have important consequences for those considering using DC and viral vectors. Furthermore, these data also suggest that DC can be significantly modulated by infection with VV-IL-2, but that the overall antigenic context, as well as individual donor features, play an important role in defining the DC phenotypic outcome.

\section{DC respond differentially to their immediate antigenic and cytokine microenvironment}

We dissected out DC responses to some of the individual components that would be found in VV preparations; that is, the cellular debris generated from the lysed cell line in which the virus was propagated, viral particles (using a noncytokine expressing control VV construct) and the cytokine itself. While there are reports showing that DC can transiently produce their own IL- $2^{45}$ which could result in DC maturation, treatment with exogenous IL-2 in this study did not induce a significant alteration in DC phenotype.

Recently, various forms of killed tumor cells have been used as a source of tumor antigens in DC vaccination protocols. However, there are conflicting data regarding the impact that killed (apoptotic versus necrotic) cells have on DC maturation and function. ${ }^{46-48} \mathrm{We}$ found that freeze-thawed lysate from 143B cells (a human osteosarcoma cell line) activated immature DC; CD80 and CD86 expression levels increased. This was confounded by HLA-DR responses that were generally downregulated, a phenomenon also seen in mature DC. Importantly, our data show that the DC phenotype induced by lysate was not retained when VV was also present.

The differential responses seen when a control VV vector, versus VV-IL-2, was applied to immature DC in the same (143B) lysate were of interest. In particular, the lysate alone increased CD86 expression, no change was seen with the control virus, and CD86 levels were decreased with VV-IL-2. These results suggest that DC may process a number of antigens simultaneously, all of which impact upon the DC, and that some antigens dominate over others in determining the eventual DC phenotype.

\section{Differential responses of mature versus immature DC}

Mature DC responses were more consistent than, but different from, those seen by immature DC after exposure to the same VV preparation. This could be because of differences in the antigenic uptake capacity of mature versus immature DC; phagocytosis of particulate antigen is more efficient in immature DC. DC maturation has also been linked to the presence of heat shock proteins (HSPs); hence chicken fibroblasts may contain higher levels of HSP than the 143B lysate. Alternatively, the foreign nature of chicken fibroblasts may be more immunostimulatory than human 143B tumor cells.

IL-12 production is another parameter of maturation, and immature DC did not produce IL-12. However, nor did DC treated with VV-IL-2 in chicken fibroblast lysate (with or without IFN $\gamma$ priming) despite evidence of maturation, although these DC did produce significant levels of IL-10 and TNF $\alpha$, indicative of a " $\mathrm{DC}_{2}$ phenotype", implying that they may induce either a Th2 response or, indeed, a tolerogenic response.

DC required signals delivered by both LPS and IFN $\gamma$ before they produced significant levels of IL-12. Interestingly, DC treated with LPS, IFN $\gamma$ and VV-IL-2 produced contrasting IL-12 responses that appeared to be determined by the cellular context within which the virus was given; that is, VV-IL-2 in chicken fibroblasts further upregulated IL-12 production, versus VV-IL-2 in 143B lysate which virtually shut down IL-12 production. The latter observation was particularly striking given that mature DC exposed to the control VV in the same 143B lysate produced detectable levels of IL-12, strongly implying that VV-IL-2 delivers a potent, dominating signal.

\section{Physiological outcome of the different DC phenotypes induced by VV-IL-2 infection}

Numerous studies suggest that DC expressing a classic immunostimulatory phenotype (i.e. high levels of CD80, CD86 and MHC class II molecules in association with IL12 production) are the DC that should be used as an anticancer agent. We found that VV-IL-2 within the human cellular lysate generally downregulated at least one of these surface molecules in both immature and mature DC, implying that this viral preparation may not be clinically useful. Whereas VV-IL-2 in a xenogeneic mammalian cellular context was better at stimulating immature DC to upregulate CD80, CD86 and MHC class II molecules, secrete IL-10 and TNF $\alpha$ but not IL-12, nonetheless they maintained their antigen presenting function.

Giving the same viral preparation to mature DC resulted in downregulation of CD86, CD86 and class II expression, while significantly upregulating IL-12 production. The physiological outcomes of these different DC phenotypes cannot be readily predicted and remains to be evaluated, although IL-12 may favor the development of a Th1 rather than a Th2 immune response. However, both Th subsets have been shown to eradicate tumors via different mechanisms ${ }^{49,50}$ and there is evidence that IL-12 is not an important factor in the immunostimulatory ability of CD40L-treated human DCs. ${ }^{51}$

In conclusion, DC responses to multiple antigens are complex and vary between donors, which may explain the inconsistent results seen in DC-based clinical trials. We show that DC are exquisitely sensitive to their local 
antigenic microenvironment, and that CD80, CD86 and MHC class II expression, as well as cytokine responses, generated by one set of antigens can be altered by other antigens. In this case, activating signals delivered to immature DC by a tumor cell lysate were altered by the presence of VV particles. Similarly, CD80, CD86 and MHC class II responses to IFN $\gamma$ and LPS could be differentially downmodulated by VV infection. Furthermore, IL-12 production was enhanced by VV-IL-2 in xenogeneic fibroblast cellular lysates, and ablated by VVIL-2, a human allogeneic tumor cell lysate. Thus, the maturation stage of the DC and the viral context (cellular lysate) determine the ultimate DC phenotype. Therefore, we suggest that researchers and clinicians proceed with caution, and that there are several caveats to consider, including (i) choosing a viral preparation that induces a DC phenotype that will induce a $\mathrm{T}$-cell response, (ii) infecting immature DC and (iii) screening individual donors. Nonetheless, immature DC can be manipulated by infection with a VV-IL-2 construct such that they are activated, secrete significant levels of IL-2, and can efficiently prime naïve T-cells.

\section{Acknowledgments}

This work was supported by grants from the NH\&MRC, SGIC and ALF. We thank Jane Allan for her advice and supply of the control vaccinia virus construct, as well as Dr Matthew Wikstrom and the Lotteries Commission WA Flow Cytometry Unit for assistance with FACs analysis.

\section{References}

1. Finke J, Ferrone S, Frey A, et al. Where have all the T-cells gone? Mechanisms of immune evasion by tumors. Immunol Today. 1999;20:158-160.

2. Marzo AL, Lake RA, Lo D, et al. Tumor antigens are constitutively presented in the draining lymph nodes. $J$ Immunol. 1999;162:5838-5845.

3. Nelson DJ, Mukherjee S, Bundell C, et al. Tumor progression despite efficient tumor antigen cross-presentation and effective "arming" of tumor antigen-specific CTL. J Immunol. 2001;166:5557-5566.

4. Mukherjee S, Haenel T, Himbeck R, et al. Replicationrestricted vaccinia as a cytokine gene therapy vector in cancer: persistent transgene expression despite antibody generation. Cancer Gene Ther. 2000;7:663-670.

5. Bernsen MR, Tang JW, Everse LA, et al. Interleukin 2 (IL-2) therapy: potential advantages of locoregional versus systemic administration. Cancer Treat Rev. 1999; 25:73-82.

6. Pantuck AJ, Belldegrun AS. Phase I clinical trial of interleukin 2 (IL-2) gene therapy for prostate cancer. Curr Urol Rep. 2001;2:33.

7. Tartour E, Mehtali M, Sastre-Garau X, et al. Phase I clinical trial with IL-2-transfected xenogeneic cells administered in subcutaneous metastatic tumours: clinical and immunological findings. Br J Cancer. 2000;83:1454-1461.
8. Dorval T, Mathiot C, Chosidow O, et al. IL-2 phase II trial in metastatic melanoma: analysis of clinical and immunological parameters. Biotechnol Ther. 1992;3:63-79.

9. Castagneto B, Zai S, Mutti L, et al. Palliative and therapeutic activity of IL-2 immunotherapy in unresectable malignant pleural mesothelioma with pleural effusion: results of a phase II study on 31 consecutive patients. Lung Cancer. 2001;31:303-310.

10. Krastev Z, Koltchakov V, Vladov N, et al. A mesothelioma that is sensitive to locally applied IL-2. Cancer Immunol Immunother. 2001;50:226-227.

11. Steinman RM, Inaba K, Turley S, et al. Antigen capture, processing, and presentation by dendritic cells: recent cell biological studies. Hum Immunol. 1999;60:562-567.

12. Huang AY, Golumbek P, Ahmadzadeh M, et al. Role of bone marrow-derived cells in presenting MHC class Irestricted tumor antigens. Science. 1994;264:961-965.

13. Bennett SR, Carbone FR, Karamalis F, et al. Induction of a $\mathrm{CD} 8+$ cytotoxic $\mathrm{T}$ lymphocyte response by cross-priming requires cognate $\mathrm{CD} 4+\mathrm{T}$-cell help. $J$ Exp Med. 1997;186:65-70.

14. Marzo AL, Lake RA, Robinson BWS, et al. T-cell receptor transgenic analysis of tumor-specific CD8 and CD4 responses in the eradication of solid tumors. Cancer Res. 1999;59:1071-1079.

15. Steinman RM, Dhodapkar M. Active immunization against cancer with dendritic cells: the near future. Int $J$ Cancer. 2001;94:459-473.

16. Nouri-Shirazi M, Banchereau J, Fay J, et al. Dendritic cell based tumor vaccines. Immunol Lett. 2000;74:5-10.

17. Celluzzi CM, Mayordomo JI, Storkus WJ, et al. Peptidepulsed dendritic cells induce antigen-specific CTL-mediated protective tumor immunity. J Exp Med. 1996;183:283-287.

18. Nestle FO, Alijagic S, Gilliet $M$, et al. Vaccination of melanoma patients with peptide- or tumor lysate-pulsed dendritic cells. Nat Med. 1998;4:328-332.

19. Zhou Y, Bosch ML, Salgaller ML. Current methods for loading dendritic cells with tumor antigen for the induction of antitumor immunity. J Immunother. 2002;25:289-303.

20. Tirapu I, Rodriguez-Calvillo M, Qian C, et al. Cytokine gene transfer into dendritic cells for cancer treatment. Curr Gene Ther. 2002;2:79-89.

21. Berard F, Blanco P, Davoust J, et al. Cross-priming of naive CD8 T-cells against melanoma antigens using dendritic cells loaded with killed allogeneic melanoma cells. J Exp Med. 2000;192:1535-1544.

22. Dhodapkar MV, Steinman RM, Krasovsky J, et al. Antigen-specific inhibition of effector T-cell function in humans after injection of immature dendritic cells. $J$ Exp Med. 2001;193:233-238.

23. Drillien R, Spehner D, Bohbot A, et al. Vaccinia virusrelated events and phenotypic changes after infection of dendritic cells derived from human monocytes. Virology 2000;268:471-481.

24. Jenne L, Hauser C, Arrighi JF, et al. Poxvirus as a vector to transduce human dendritic cells for immunotherapy: abortive infection but reduced APC function. Gene Therapy. 2000;7:1575-1583.

25. Engelmayer J, Larsson M, Subklewe M, et al. Vaccinia virus inhibits the maturation of human dendritic cells: a novel mechanism of immune evasion. J Immunol. 1999;163:67626768.

26. Drexler I, Antunes E, Schmitz M, et al. Modified vaccinia virus ankara for delivery of human tyrosinase as melanomaassociated antigen: induction of tyrosinase- and melanoma- 
specific human leukocyte antigen a*0201-restricted cytotoxic T-cells in vitro and in vivo. Cancer Res. 1999;59:49554963.

27. Kim CJ, Cormier $\mathbf{J}$, Roden $\mathbf{M}$, et al. Use of recombinant poxviruses to stimulate anti-melanoma T-cell reactivity. Ann Surg Oncol. 1998;5:64-76.

28. Yang S, Kittlesen D, Slingluff Jr. CL, et al. Dendritic cells infected with a vaccinia vector carrying the human gp100 gene simultaneously present multiple specificities and elicit high-affinity $\mathrm{T}$ cells reactive to multiple epitopes and restricted by HLA- $\alpha 2$ and $-\alpha 3$. J Immunol. 2000;164:42044211.

29. Kedl RM, Rees WA, Hildeman DA, et al. T-cells compete for access to antigen-bearing antigen-presenting cells. $J$ Exp Med. 2000;192:1105-1113.

30. Norbury CC, Malide D, Gibbs JS, et al. Visualizing priming of virus-specific CD8 + T-cells by infected dendritic cells in vivo. Nat Immunol. 2002;3:265.

31. Nossal GJ. Tolerance and ways to break it. Ann NY Acad Sci. 1993;690:34-41.

32. Tham EL, Shrikant P, Mescher MF. Activation-induced nonresponsiveness: a Th-dependent regulatory checkpoint in the CTL response. $J$ Immunol. 2002;168:1190-1197.

33. Romani N, Gruner S, Brang D, et al. Proliferating dendritic cell progenitors in human blood. J Exp Med. 1994;180:8393.

34. Ramshaw IA, Andrew ME, Phillips SM, et al. Recovery of immunodeficient mice from a vaccinia virus/IL-2 recombinant infection. Nature. 1987;329:545-546.

35. Warren HS, Kinnear BF, Skipsey LJ. Human natural killer (NK) cells: requirements for cell proliferation and expansion of phenotypically novel subpopulations. Immunol Cell Biol. 1993;71:87-97.

36. Brossart P, Goldrath AW, Butz EA, et al. Virus-mediated delivery of antigenic epitopes into dendritic cells as a means to induce CTL. J Immunol. 1997;158:3270-3276.

37. Di Nicola $M$, Siena $S$, Bregni $M$, et al. Gene transfer into human dendritic antigen-presenting cells by vaccinia virus and adenovirus vectors. Cancer Gene Ther. 1998; 5:350-356.

38. Kim CJ, Prevette T, Cormier J, et al. Dendritic cells infected with poxviruses encoding MART-1/melan A sensitize $\mathrm{T}$ lymphocytes in vitro. J Immunother. 1997;20:276-286.

39. Bronte V, Carroll MW, Goletz TJ, et al. Antigen expression by dendritic cells correlates with the therapeutic effectiveness of a model recombinant poxvirus tumor vaccine. Proc Natl Acad Sci USA. 1997;94:3183-3188.

40. Akiyama Y, Watanabe M, Maruyama K, et al. Enhancement of antitumor immunity against B16 melanoma tumor using genetically modified dendritic cells to produce cytokines. Gene Therapy. 2000;7:2113-2121.

41. Shimizu K, Fields RC, Giedlin M, et al. Systemic administration of interleukin 2 enhances the therapeutic efficacy of dendritic cell-based tumor vaccines. Proc Natl Acad Sci USA. 1999;96:2268-2273.

42. Shimizu K, Fields RC, Redman BG, et al. Potentiation of immunologic responsiveness to dendritic cell-based tumor vaccines by recombinant interleukin-2. Cancer J Sci Am. 2000;6(Suppl 1) :S67-S75

43. Eggert AO, Becker JC, Ammon M, et al. Specific peptidemediated immunity against established melanoma tumors with dendritic cells requires IL-2 and fetal calf serum-free cell culture. Eur J Immunol. 2002;32:122-127.

44. Faulkner L, Buchan G, Lockhart E, et al. IL-2 linked to a peptide from influenza hemagglutinin enhances T-cell activation by affecting the antigen-presentation function of bone marrow-derived dendritic cells. Int Immunol. 2001;13:713-721.

45. Granucci F, Vizzardelli C, Pavelka N, et al. Inducible IL-2 production by dendritic cells revealed by global gene expression analysis. Nat Immunol. 2001;2:882-888.

46. Sauter B, Albert ML, Francisco L, et al. Consequences of cell death: exposure to necrotic tumor cells, but not primary tissue cells or apoptotic cells, induces the maturation of immunostimulatory dendritic cells. $J$ Exp Med. 2000;191:423-434.

47. Ferlazzo G, Semino C, Spaggiari GM, et al. Dendritic cells efficiently cross-prime HLA class I-restricted cytolytic T lymphocytes when pulsed with both apoptotic and necrotic cells but not with soluble cell-derived lysates. Int Immunol. 2000;12:1741-1747.

48. Kotera Y, Shimizu K, Mule JJ. Comparative analysis of necrotic and apoptotic tumor cells as a source of antigen(s) in dendritic cell-based immunization. Cancer Res. 2001;61:8105-8109.

49. Fernando GJP, Stewart TJ, Tindle RW, et al. Th2-type CD4 + cells neither enhance nor suppress antitumor CTL activity in a mouse tumor model. J Immunol. 1998;161:24212427.

50. Nishimura $T$, Iwakabe $K$, Sekimoto $M$, et al. Distinct role of antigen-specific $\mathrm{T}$ helper type 1 (Th1) and Th2 cells in tumor eradication in vivo. J Exp Med. 1999;190: 617-627.

51. Kuniyoshi JS, Kuniyoshi CJ, Lim AM, et al. Dendritic cell secretion of IL-15 is induced by recombinant huCD40Lt and augments the stimulation of antigen-specific cytolytic Tcells. Cell Immunol. 1999;193:48-58. 